\title{
Calidad fisicoquímica y microbiológica del agua superficial del río Grande de Tárcoles, Costa Rica: un enfoque ecológico
}

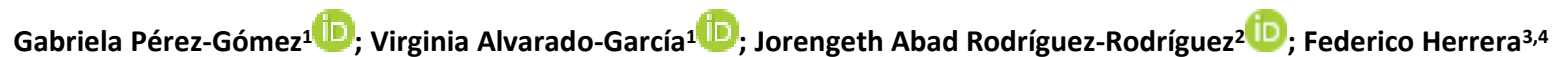 \\ (iD) $y$ Rolando Sánchez-Gutiérrez ${ }^{5}$ iD
}

1. Universidad Estatal a Distancia, Vicerrectoría de Investigación, Laboratorio de Vida Silvestre y Salud, 2050 Sabanilla, San José, Costa Rica; gperez@uned.ac.cr; valvaradog@uned.ac.cr

2. Universidad Nacional, Escuela de Ciencias Biológicas, Laboratorio de Biotecnología Microbiana, Costa Rica; jorengeth.rodriguez.rodriguez@una.cr

3. Universidad Estatal a Distancia, Escuela de Ciencias Exactas y Naturales, Sabanilla, San José, Costa Rica; fherreram@uned.ac.cr

4. Universidad Nacional, Escuela de Ciencias Biológicas, Laboratorio de Enseñanza de las Ciencias, Heredia, Costa Rica.

5. Universidad Nacional, Escuela de Química, Laboratorio de Manejo del Recurso Hídrico y Grupo de Investigación en Isotopos Estables, Costa Rica; rolando.sanchez.gutierrez@una.ac.cr

Recibido 04-IX-2020 • Corregido 22-XI-2020 • Aceptado 26-XI-2020

DOI: https://doi.org/10.22458/urj.v13i1.3148

\begin{abstract}
Physicochemical and microbiological quality of surface water in the Grande de Tárcoles River, Costa Rica: an ecological approach". Introduction: The quality of the water in a hydrographic basin and its potential use are affected by anthropic and natural factors. The Tárcoles River is the final receiver of waters from the central region of Costa Rica, where the treatment of wastewater is inadequate or non-existent. Objective: To evaluate water pollution in the river and review macroinvertebrates as ecosystem bioindicators. Methods: Physicochemical, microbiological and macroinvertebrate evaluations of the surface water were done in four sites for twelve consecutive months, including two water quality indices (ICA-NSF and Dutch). Monthly visits were made for the placement and collection of artificial substrates for aquatic macroinvertebrates, and bimonthly visits for the collection of water samples for ex situ analysis of the physicochemical and microbiological parameters. In addition, the land use in the lower part of Tárcoles was characterized from cover raster files. Results: Some physicochemical variables showed similar concentrations to those reported for other Costa Rican rivers, while oxygen showed lower values. The microbiological variables indicated high pollution levels, especially in the rainy season. In the dry season, there were more aquatic macroinvertebrates, and Chironomidae, Oligochaeta and Hydrobiidae were the most abundant. River pollution is categorized as "incipient" according to the Dutch index and as "medium quality" according to the ICA-NSF. Conclusions: the pollution of the Tárcoles River is mainly due to inadequate land use and anthropic activities. We recommend a basin management plan and research on ecosystem health and conservation of vulnerable species.

Keywords: Bioindicators, water quality, Grande de Tárcoles River, aquatic macroinvertebrates, physicochemical parameters.
\end{abstract}

RESUMEN. Introducción: La calidad del agua en una cuenca hidrográfica, y su uso potencial, se ven afectados por factores antrópicos y naturales. El río Tárcoles es el receptor final de aguas de la región central de Costa Rica, donde el tratamiento de aguas residuales es inadecuado o inexistente. Objetivo: Evaluar la contaminación del agua en el río y evaluar a los macroinvertebrados como bioindicadores del ecosistema. Métodos: Se realizaron evaluaciones fisicoquímicas, microbiológicas y de macroinvertebrados del agua superficial en cuatro sitios durante doce meses consecutivos, incluidos dos índices de calidad del agua (ICA-NSF y holandés). Hicimos visitas mensuales para la colocación y recolección de sustratos artificiales para macroinvertebrados acuáticos, y visitas bimensuales para la recolección de muestras de agua para análisis ex situ de los parámetros fisicoquímicos y microbiológicos. Además, se caracterizó el uso del suelo en la parte baja de Tárcoles a partir de archivos tipo "raster" de cobertura. Resultados: Algunas variables fisicoquímicas tuvieron concentraciones similares a las informadas para otros ríos costarricenses, mientras que el oxígeno fue inferior. Las variables microbiológicas indicaron altos niveles de contaminación, especialmente en la época de lluvias. En la estación seca, hubo más macroinvertebrados acuáticos, siendo Chironomidae, Oligochaeta e Hydrobiidae los más abundantes. La contaminación de los ríos se clasifica como "incipiente" según el índice holandés y como "calidad media" según el ICA-NSF. Conclusiones: la contaminación del río Tárcoles se debe principalmente a un uso inadecuado del suelo y actividades antrópicas. Recomendamos un plan de gestión de cuencas e investigación sobre la salud del ecosistema y la conservación de especies vulnerables.

Palabras clave: Bioindicadores, calidad del agua, Grande de Tárcoles, macroinvertebrados acuáticos, parámetros fisicoquímicos. 
La cuenca del río Grande de Tárcoles representa el 4,2\% de la superficie del país, concentra el 55\% de la población (Espinoza \& Villalta, 2004; Meléndez \& Russo 2016) y el 80\% de la industria (Blomquist, Ballestero, Bhat, \& Kemper, 2007). Se considera como la cuenca más contaminada de Centroamérica (DFOE, 2013), porque la mayor parte de las aguas residuales e industriales, no reciben tratamiento o es inadecuado (Espinoza \& Villalta, 2004; Chamizo \& Mora, 2006; CalvoBrenes \& Mora-Molina, 2007). En el año 2015 se inauguró la "Planta de Tratamiento los Tajos", que recibe una parte de las aguas residuales de San José (Presidencia de la República, 2016), sin embargo, el resto de la Gran Área Metropolitana (GAM) de Costa Rica permanece sin tratamiento adecuado (Meléndez \& Russo, 2016).

El Tárcoles es el receptor final de las aguas provenientes de la región central del país, con vertidos como: residuos sólidos, nutrientes, metales pesados, sustancias químicas orgánicas, plaguicidas, hidrocarburos, grasas, aceites, entre otros (Espinoza \& Villalta, 2004; Chamizo \& Mora, 2006; Blomquist et al., 2007; Calvo-Brenes \& Mora-Molina, 2007; DFOE, 2013; Meléndez \& Russo, 2016). La calidad del agua de una cuenca hidrográfica y su uso potencial, se ve afectado por la interacción de factores antrópicos y naturales (Gastezzi-Arias, Alvarado-García \& Pérez-Gómez, 2017). Por lo tanto, los valores de referencia para la calidad del agua pueden ser establecidos, a través del monitoreo de los parámetros físicos, químicos y biológicos (Aznar, 2000; Pérez, Segovia, Cabrera, Delgado, \& Martins, 2018) y, para esto se deben integrar índices de calidad robustos que permitan un resultado integral (Pérez-Castillo \& Rodríguez, 2008; Mena-Rivera et al., 2018). Un ejemplo de ello, es el índice de calidad del agua (ICA), establecido por la Fundación Nacional de Saneamiento (NSF, Dallas, Estados Unidos). Este se basa en nueve parámetros y ha sido ampliamente utilizado (Samboni, Carvajal, \& Escobar, 2007; Zhen-Wu, 2010; Mena-Rivera et al., 2018).

En Costa Rica, este índice se ha utilizado en ríos urbanos, tales como, río Tibás, río Uruca, río Pirro, río Burío, río Cañuela, río Virilla, entre otros (Coto, Benavides \& Segura, 2008; Gurdián \& Coto 2010; Pérez et al., 2012; Castillo, Coto, \& Herrera, 2012; Blanco \& Morera, 2013; Herrera, Rodríguez, Rojas, Herrera, \& Chaves, 2013; Benavides-Benavides et al., 2016; Mena-Rivera, SalgadoSilva, Benavides-Benavides, Coto-Campos, \& Swinscoe, 2017; Pérez-Gómez, Jiménez-Rocha, \& Bermúdez-Rojas, 2018; Mena-Rivera et al., 2018). Como complemento a estos parámetros fisicoquímicos, se incluyen variables biológicas, como los macroinvertebrados acuáticos y el análisis microbiológico. Basados en metodologías más robustas que muestran tendencias a largo plazo y permiten detectar eventos puntuales de toxicidad (Samboni et al., 2007; Springer, 2010).

Los macroinvertebrados acuáticos son considerados excelentes bioindicadores de la calidad del agua, debido a su tamaño, su amplio rango de distribución, su adaptación a diferentes variables físico-bióticas (Lozano, 2005; Prat, Ríos, Acosta, \& Rieradevall, 2009) y su alta sensibilidad a alteraciones del medio (Forero-Céspedes, Reinoso-Flórez, \& Gutiérrez, 2013). Sin embargo, las metodologías tradicionales por captura directa no son recomendables en ríos de difícil acceso (La Gaceta, 2007), profundos y caudalosos como el Tárcoles, que además posee una alta contaminación hídrica, profundidad y riesgo por poblaciones de cocodrilos (Ramírez, 2010; Mauger et al., 2012). Debido a lo anterior, se recomienda utilizar metodologías alternas y económicas, como el uso de sustratos artificiales, que puedan ser colonizados por los macroinvertebrados acuáticos, considerando que el tipo de sustrato presente en el lecho del río es un factor de alta importancia para determinar la naturaleza y estructura de la comunidad bentónica (Quesada-Alvarado \& SolanoUlate, 2020).

Por otro lado, las enterobacterias se encuentran ampliamente distribuidas a nivel mundial, una de ellas, Escherichia coli, es de gran importancia como indicador de contaminación por materia fecal en cuerpos de agua, ya que habitan en el intestino del ser humano, animales de pastoreo, 
reptiles y aves; actuando éstos como reservorios en la cadena de transmisión. Puntualmente, el riesgo sanitario causado por los microorganismos se incrementa en las partes medias y bajas de la cuenca lo que limita su uso para las actividades productivas y se vuelve un problema ecológico (Mena-Rivera et al., 2018), es así como, las actividades humanas aceleran la proliferación de enfermedades asociadas al recurso hídrico (Pastor, Varela, Bianchi, \& Durando, 2015).

Es por esto, que esta investigación pretende evaluar el estado de contaminación del agua superficial del Tárcoles, mediante la aplicación de dos índices de calidad de agua, así como una revisión ecológica de la comunidad de macroinvertebrados como bioindicadores de la salud de este ecosistema, a través del uso de sustratos artificiales.

\section{MATERIALES Y MÉTODOS}

Sitio de estudio: La cuenca del río Grande de Tárcoles se ubica en el sector central y occidental del Valle Central de Costa Rica y se extiende hasta la Vertiente del Pacífico. Está conformada por la confluencia de dos subcuencas: el río Virilla $\left(829 \mathrm{~km}^{2}\right)$ y el río Grande de San Ramón $\left(916 \mathrm{~km}^{2}\right.$ ) (Quesada, 2012). Cubre un área de $2155 \mathrm{~km}^{2}$, posee una longitud del cauce de $111 \mathrm{~km}$; se encuentra a una elevación entre los 0 y los 2960 metros sobre el nivel del mar (m.s.n.m.), la temperatura oscila entre los 15 a $33^{\circ} \mathrm{C}$ y la precipitación media anual varía entre 948 a los $5409 \mathrm{~mm}$ (Blomquist et al., 2007).

Se trabajó en la parte baja de la cuenca, en el cauce principal del Tárcoles, el cual comprende una superficie de $488 \mathrm{~km}^{2}$, cubierta por bosque secundario, cultivos y pastos, bosque maduro, suelo desnudo, urbano, manglares, agua y humedales (Sánchez, 2017). Se identificaron cuatro sitios de estudio (Fig. 1). El primero se ubicó dentro del Proyecto Hidroeléctrico Chucás, en Atenas, específicamente dentro del embalse $\left(9^{\circ} 55^{\prime} 55.60^{\prime \prime} \mathrm{N}, 84^{\circ} 22^{\prime} 58.86^{\prime \prime O}\right)$; el segundo se ubicó en el Puente de Hamacas que divide los cantones de Turrubares y Orotina ( $\left.9^{\circ} 52^{\prime} 48.18^{\prime \prime} \mathrm{N}, 84^{\circ} 31^{\prime} 40.04^{\prime \prime O}\right)$; el tercero fue en el Tajo La Garita, ubicado cerca del Parque Nacional Carara $\left(9^{\circ} 48^{\prime} 59.62^{\prime \prime} \mathrm{N}\right.$, $\left.84^{\circ} 34^{\prime} 59.43 " 0\right) ;$ y el último a $3 \mathrm{~km}$ de la desembocadura del Tárcoles, en el pueblo de Tárcoles (946'39.42"N, 84³7'32.68"O).

Diseño de muestreo: Se caracterizó el uso de suelo en la parte baja de la cuenca del Tárcoles, a partir del archivo ráster de cobertura para Costa Rica (Sánchez, 2017). Se realizaron visitas mensuales para la colocación y recolecta de sustratos artificiales de macroinvertebrados acuáticos; la valoración de parámetros ambientales; y el registro de parámetros fisicoquímicos del agua in situ. Además, se realizaron visitas bimensuales para la recolecta de muestras de agua para los análisis ex situ de parámetros fisicoquímicos y microbiológicos. El período de estudio abarcó 12 meses (diciembre 2017 a diciembre 2018) y dos épocas: seca (diciembre - mayo) y lluviosa (junionoviembre). Para la recolección de muestras microbiológicas, fisicoquímicas y sustratos artificiales, se siguieron los protocolos establecidos en el Reglamento de Evaluación y Clasificación de la Calidad de Cuerpos de Agua Superficiales № 33903-MINAE-S (La Gaceta, 2007), así como el método estándar (SM) 1060 (APHA, AWWA, \& WEF, 2017). Además, se tomaron en cuenta modificaciones al método de recolecta de macroinvertebrados (Quesada-Alvarado \& Solano-Ulate, 2020). 


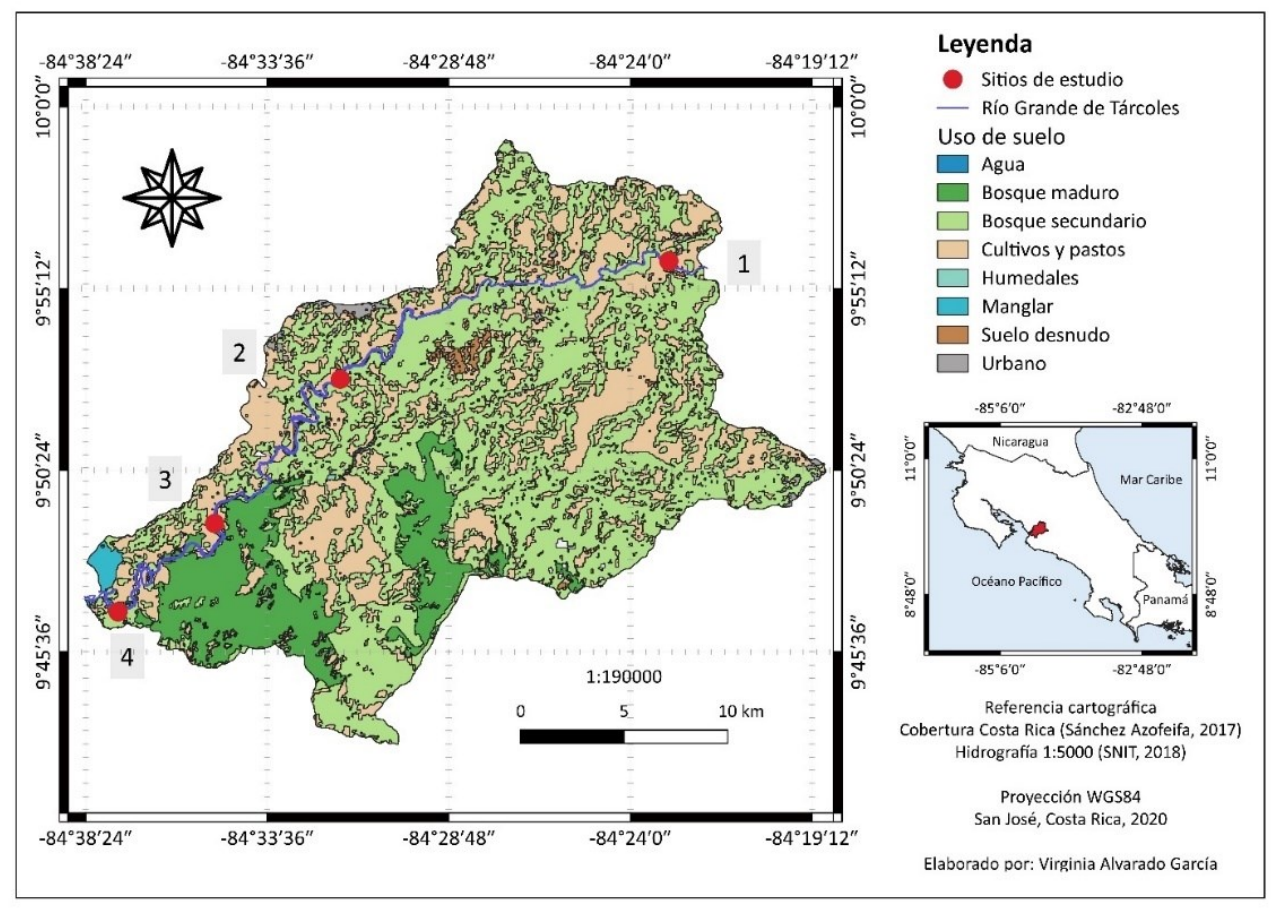

Fig. 1. Uso de suelo y sitios de estudio, (1): Planta Hidroeléctrica Chucás, Atenas, (2): Puente de Hamacas, Orotina, (3): Tajo La Garita, Turrubares, (4) Desembocadura, Jungle Crocodile Safari, Tárcoles.

Parámetros físicos y químicos: Las muestras se recolectaron en recipientes de polietileno de alta densidad previamente acondicionados y se mantuvieron a $6^{\circ} \mathrm{C}$ o menos. Se trasladaron al laboratorio en un periodo menor a 24 horas, donde permanecieron resguardadas en las mismas condiciones. La temperatura, conductividad, sólidos disueltos totales (SDT) oxígeno disuelto (OD), porcentaje de saturación de oxígeno (PSO), pH y potencial de oxidación reducción (ORP), fueron medidas en los sitios de muestreo utilizando un medidor multiparamétrico Hanna Instruments HI98194 (Limena, Italia). La turbidez se midió en el laboratorio mediante la técnica neftelométrica (SM 2130) utilizando un medidor Oakton T100 (Vernon Hils, IL, USA); la demanda bioquímica de oxígeno $\left(\mathrm{DBO}_{5}{ }^{20}\right)$ se analizó mediante el método de electrodo de membrana (SM 4500-O. G), utilizando una sonda óptica YSI ProOBOD (Ohio, USA); los sólidos totales (ST) mediante gravimetría (SM 2540 B); el análisis de los cationes y aniones (calcio, magnesio, sodio, potasio, fluoruro, cloruro, bromuro, nitrato y sulfato) se realizó mediante cromatografía de iones (SM 4110), utilizando un cromatógrafo ThermoScientific ICS 5000+EG (Sunnyvale, CA, USA); el ión amonio se analizó mediante espectrofotometría visible utilizando el método del azul de indofenol (SM 4500-NH3 F); el fósforo se midió como fósforo total (FT) empleando la digestión con persulfato de amonio y el método espectrofotométrico de cloruro de estaño (SM 4500-P. B y D).

Parámetros microbiológicos (coliformes totales y fecales): Las muestras se recolectaron en frascos estériles de $100 \mathrm{~mL}$ rotulados previamente y se mantuvieron a $4^{\circ} \mathrm{C}$ hasta su traslado al laboratorio. En el campo la muestra fue tomada introduciendo en el agua el frasco tapado (para evitar contaminación externa) y se abrió lentamente bajo el agua para la toma de la muestra, finalmente se cerró debajo del agua.

Para el análisis, se hicieron diluciones seriadas base diez, desde $10^{-1}$ hasta $10^{-9}$ a cada muestra, en agua peptonada bufferizada estéril marca Difco a pH 7,2 y luego se procedió a usar la técnica de fermentación de tubos múltiples por serie de cinco con medio Fluorocult ${ }^{\circledR}$ a 
concentración sencilla e incubados $24-48 \mathrm{~h}$ a $37^{\circ} \mathrm{C}$ (Bartram \& Ballance, 1996; Merck, 2005). La lectura de los tubos positivos para coliformes totales se realizó enumerando la cantidad de tubos que cambiaron de color por cada nivel de dilución; mientras que, los coliformes fecales se evidenciaron al agregar reactivo de Ehrlich a los tubos que cambiaron de color, y se tomaron como positivos los que desarrollaron un anillo color rojo (Merck, 2005). La determinación de la carga microbiana se realizó utilizando la tabla de "Número más probable" (NMP) para cinco tubos (Woomer, 1994).

Parámetros biológicos - Macroinvertebrados: En cada sitio de estudio se colocaron de forma separada tres tipos diferentes sustratos artificiales, conteniendo hojas: 1- hojas, 2- trozos de madera y 3 - rocas. Aproximadamente, $50 \mathrm{~m}$ antes y $50 \mathrm{~m}$ después de la colocación del primer grupo formado por los tres tipos de sustratos, se colocó una réplica de cada tipo de sustrato artificial. Por lo tanto, por cada sitio, se colocaron un total de nueve sustratos artificiales tratando de abarcar la mayor parte de los microhábitats presentes (Quesada-Alvarado \& Solano-Ulate, 2020). Cada sustrato se elaboró con malla estándar plástica de $1 \mathrm{~cm}$ de luz, cerrada en uno de sus extremos formando una bolsa en cuyo interior se colocó sólo uno de los tres tipos de sustratos, y posteriormente se cerró el otro extremo de la bolsa. En el caso de los sustratos artificiales con hojas, se colocaron $30 \mathrm{~g}$ de hojas de vegetación ribereña circundante y abundante. Se utilizaron hojas frescas principalmente de Balsa (Ochroma pyramidale) y, en una proporción menor al $10 \%$, hojas frescas de Guácimo (Guazuma ulmifolia) y Bateita (Amphilophium crucigerum). Mientras que, para los sustratos artificiales con piedra, se utilizaron $170 \mathrm{~g}$ que no sobrepasó los $15 \mathrm{~cm}$ de diámetro. Tanto las hojas, como las piedras, fueron obtenidas en el margen cercano al río y no se les aplicó ningún tratamiento. Finalmente, para los sustratos artificiales con madera, se utilizó entre 130 y $140 \mathrm{~g}$ principalmente de Jaúl (Alnus acuminata) recién cortado y sin ningún tratamiento previo a la colocación de los sustratos. Los sustratos artificiales fueron colocados sobre el fondo y en las orillas a lo largo del cauce del río y debidamente fijados con una cuerda de nylon, para evitar ser arrastrados por crecidas o la misma corriente del río. Los sustratos permanecieron sumergidos por 30 días (La Gaceta, 2007). Una vez recolectados se preservaron en etanol al 95\% y fueron transportados al laboratorio donde fueron procesados. Se utilizaron tres tamices, en donde el menor fue de $500 \mu \mathrm{m}$ de luz. La identificación taxonómica de los especímenes llegó hasta el nivel de familia, en la mayoría de los casos, utilizando un estereoscopio y claves taxonómicas especializadas (Vargas, 1974; Roldán, 1988; Merritt, Cummins, \& Berg, 2008; Springer, Ramírez, \& Hanson, 2010). Posteriormente, los organismos identificados fueron etiquetados, conservados en etanol al $70 \%$ y depositados en la Colección Geográfica de Entomología Acuática del Museo de Zoología de la Universidad de Costa Rica.

Índices de Calidad de Agua (ICA): Se calcularon dos ICA, el primero corresponde al índice holandés de valoración de calidad del agua para cuerpos receptores, el cual se encuentra normado en el decreto N³3903- MINAE-S, "Reglamento para la Evaluación y Clasificación de la Calidad de Cuerpos de Agua Superficiales" (RECAAS) (La Gaceta, 2007), que contempla tres parámetros: PSO (\%), $\mathrm{DBO}(\mathrm{mg} / \mathrm{L})$ y N-NH${ }_{4}^{+}(\mathrm{mg} / \mathrm{L})$, y el segundo fue el índice de la fundación nacional de saneamiento de los Estados Unidos de América (Brown, McClelland, Deininger, \& Tozer, 1970; Oram, 2013), el cual valora nueve parámetros: DBO $(\mathrm{mg} / \mathrm{L})$, PSO (\%), coliformes fecales (NMP/100mL), nitratos $(\mathrm{mg} / \mathrm{L}), \mathrm{pH}$, temperatura $\left({ }^{\circ} \mathrm{C}\right), \mathrm{SDT}(\mathrm{mg} / \mathrm{L}), \mathrm{FT}(\mathrm{mg} / \mathrm{L})$ y turbidez (UNT).

Análisis de resultados: Se realizó la estadística descriptiva para todos los parámetros analizados, aplicando la técnica de regresión en orden estadístico (ROS) para calcular el promedio y la desviación estándar de aquellos parámetros donde se obtuvieron valores menores al límite de cuantificación de la técnica analítica empleada con excepción de las variables que cumplieran esta 
característica en más del $80 \%$ de los datos (Helsel, 2012). Adicionalmente, se realizó un análisis estadístico más exhaustivo, aplicando pruebas de contraste de medianas a través de la prueba de Kruskal-Wallis (Kruskal \& Wallis, 1952) con el objetivo de identificar diferencias significativas en las variables medidas por sitios y épocas, para el caso de las variables con valores menores al límite de cuantificación donde esta característica no supera el $80 \%$ de los datos, se aplicó la prueba de inferencia de Peto-Prentice (Peto \& Peto, 1972; Prentice \& Markel, 1979). Además, se realizó un análisis multivariado de componentes principales para comprender el grado de variación que se logra explicar en el conjunto de datos a través de los aportes relativos, de las variables medidas, dentro de cada componente principal. Los análisis estadísticos se realizaron mediante el lenguaje de programación R 3.6.2, R Core Team, (2020), utilizando paquetes como ggplot2 (Wickham, 2016), factoextra (Kassambara, 2015), asbio (Aho,2014) y NADA (Lee, 2017).

\section{RESULTADOS}

Se identificaron ocho usos de suelo en la parte baja de la cuenca. Los más predominantes fueron, el bosque secundario (51,99\%), cultivos y pastos $(31,07 \%)$, y bosque maduro $(12,76 \%)$. El suelo desnudo y el urbanismo no superaron el $2 \%$; mientras que, los manglares, el agua y los humedales, se mantuvieron por debajo del $1 \%$. Además, durante los muestreos realizados en los años 2017 y 2018 se registró en promedio, una temperatura de $28,48^{\circ} \mathrm{C}$, una humedad relativa de $72 \%$ y una velocidad del viento de $3,45 \mathrm{~m} / \mathrm{s}$.

Parámetros físicos, químicos y microbiológicos: Las variables físicas como temperatura, contenido de sólidos, conductividad, $\mathrm{pH}, \mathrm{ORP}$ y turbidez, son congruentes con las concentraciones reportadas para otros sistemas lóticos en Costa Rica. Sin embargo, el porcentaje y la saturación de saturación de oxígeno en el sitio 1 , mostraron valores significativamente menores $(\chi 2>14,2 ; p<0,05$ y $\chi 2>14,4 ; p<0,05$ respectivamente) en comparación con los demás sitios; así mismo, la temperatura para los sitios 1 y 2 presentó valores significativamente menores $(\chi 2>11,3 ; p<0,05)$. En relación con la estacionalidad, únicamente la turbidez presentó diferencia significativa para este factor $(\chi 2>8,6$; $p<0,05)$ siendo la época lluviosa la estación con valores más altos (Cuadro 1).

En el caso de las variables químicas: DBO, amonio, nitrato y el fósforo total, son los principales indicadores de contaminación por actividades antropogénicas, con concentraciones promedio típicos de aguas superficiales y niveles de contaminación incipientes y/o moderados (Cuadro 1); además, no se evidenció diferencia estadísticamente significativa entre sitios o épocas, únicamente en el caso del ion amonio para el cual las concentraciones en los sitios 1 y 2 fueron significativamente superiores $(x 2>8,2 ; p<0,05)$.

El sulfato, cloruro, sodio, potasio, calcio y magnesio presentaron altas desviaciones estándar; únicamente el sulfato no presentó diferencia estadísticamente significativa $(p>0,05)$ entre los sitios o las épocas. El bromuro y fluoruro, en más del $80 \%$ de las muestras, tuvieron concentraciones menores a los límites de cuantificación de la técnica analítica empleada $(0,786 \mathrm{mg} / \mathrm{L}$ y $0,32 \mathrm{mg} / \mathrm{L}$, respectivamente), destacando el sitio 4 con el valor máximo para el bromuro (Cuadro 1).

En cuanto a los valores de coliformes fecales y totales, las concentraciones obtenidas evidencian contaminación microbiológica en todos los sitios; sin embargo, el análisis estadístico no indicó diferencia significativa entre los sitios muestreados, más sí entre épocas, donde los coliformes son significativamente mayores en la época lluviosa $(\chi 2>13,3 ; p<0,05)$. Además, el promedio de coliformes fecales es de $27700 \mathrm{NMP} / 100 \mathrm{~mL}$ por lo que se ubica como clase 5 en el Reglamento de Evaluación y Clasificación de la Calidad de Cuerpos de Agua Superficiales № 33903-MINAE-S, como agua no utilizable en ninguna categoría (Cuadro 1). 


\section{CUADRO 1}

Resumen de las características físicas, químicas y microbiológicas del agua superficial en el Río Tárcoles.

\begin{tabular}{|c|c|c|c|c|c|}
\hline Parámetro & Unidades & Promedio & $\mathrm{DE}$ & Mínimo & Máximo \\
\hline Temperatura & ${ }^{\circ} \mathrm{C}$ & 26,7 & 1,6 & 24,0 & 30,0 \\
\hline $\mathrm{pH}$ & - & 7,61 & 0,35 & 7,05 & 8,59 \\
\hline Conductividad & $\mu \mathrm{S} / \mathrm{cm}$ & 464 & 876 & 150 & 4521 \\
\hline SDT & $\mathrm{mg} / \mathrm{L}$ & 234 & 438 & 75 & 2260 \\
\hline ST & $\mathrm{mg} / \mathrm{L}$ & 700 & 1168 & 258 & 6115 \\
\hline Turbidez & UNT & 83 & 102 & 3 & 319 \\
\hline ORP & $\mathrm{mV}$ & 183 & 45 & 84 & 271 \\
\hline DO & $\%$ & 72,6 & 21,4 & 23,1 & 116,1 \\
\hline DBO* & $\mathrm{mg} / \mathrm{L}$ & 2,52 & 1,22 & $<2,00$ & 6,15 \\
\hline Amonio* & $\mathrm{mg} / \mathrm{L} \mathrm{NH}_{4}^{+}$ & 0,132 & 0,219 & $<0,050$ & 0,862 \\
\hline Nitrato & $\mathrm{mg} / \mathrm{L} \mathrm{NO}_{3}^{-}$ & 9,7 & 7,8 & 1,8 & 43,2 \\
\hline FT & $\mathrm{mg} / \mathrm{L} \mathrm{P}-\mathrm{PO}_{4}{ }^{3-}$ & 0,371 & 0,320 & 0,100 & 1,762 \\
\hline Cloruro & $\mathrm{mg} / \mathrm{L}$ & 69,7 & 280,3 & 2,62 & 1384 \\
\hline Sulfato & $\mathrm{mg} / \mathrm{L}$ & 23,5 & 40,2 & 9,6 & 209,6 \\
\hline Fluoruro** & $\mathrm{mg} / \mathrm{L}$ & - & - & $<0,18$ & $<0,32$ \\
\hline Bromuro** & $\mathrm{mg} / \mathrm{L}$ & - & - & $<0,786$ & 5,06 \\
\hline Calcio & $\mathrm{mg} / \mathrm{L}$ & 21,8 & 7,8 & 13,2 & 53,0 \\
\hline Magnesio & $\mathrm{mg} / \mathrm{L}$ & 10,4 & 18,7 & 4,00 & 97,7 \\
\hline Sodio & $\mathrm{mg} / \mathrm{L}$ & 44,6 & 151,2 & 5,5 & 753,4 \\
\hline Potasio & $\mathrm{mg} / \mathrm{L}$ & 4,16 & 4,95 & 1,21 & 27,05 \\
\hline Coliformes totales & $\mathrm{NMP} / \mathrm{mL}$ & $3,42 \times 10^{4}$ & $5,68 \times 10^{4}$ & $2,30 \times 10^{2}$ & $2,30 \times 10^{5}$ \\
\hline Coliformes fecales & $\mathrm{NMP} / \mathrm{mL}$ & $2,77 \times 10^{4}$ & $5,27 \times 10^{4}$ & $2,10 \times 10^{2}$ & $2,30 \times 10^{5}$ \\
\hline
\end{tabular}

Notas: * Estimaciones del promedio y desviación estándar (DE) usando el método ROS; ${ }^{* *}>80 \%$ de los datos son menores al límite de cuantificación.

El 78,3\% de la variación de los datos es explicada con cinco componentes principales (fig. 2a). En el aporte de las variables para los dos primeros componentes principales no se presenta una variación con respecto al factor estacional (fig. 2b).

En el primer componente, los coliformes fecales y totales, el sodio, el magnesio, el calcio, el cloruro (iones mayoritarios del agua) y la temperatura presentan las mayores contribuciones en varianza; mientras que, en el segundo componente principal, las variables que mayor varianza aportan corresponden al nitrato, los sólidos totales, la DBO y el pH (fig. $2 \mathrm{~b}$ ).

Parámetros biológicos - Macroinvertebrados: Se obtuvo un total de 2502 individuos pertenecientes a 53 taxones (44 familias de macroinvertebrados y 9 taxones superiores a familia) (Cuadro 3, Apéndice digital).

De éstos, los que presentaron mayor número de individuos fueron: Chironomidae con un 36,53\% (914 individuos), Oligochaeta 18,23\% (456 individuos), Hydrobiidae 11,79\% (295 individuos), Physidae 6,71\% (168 individuos) y Psychodidae 4\% (100 individuos), las demás familias tuvieron una abundancia inferior a 85 individuos. Con respecto a los sitios de muestreo, la familia Chironomidae fue la más abundante en los sitios 1 y 2; Oligochaeta en el sitio 3 e Hydrobiidae en el sitio 4 .

Por otra parte, la abundancia de individuos recolectados en época seca fue la única diferencia estadística significativa que se presentó $(\chi 2>8,2 ; p<0,05)$ (Fig. 3). 

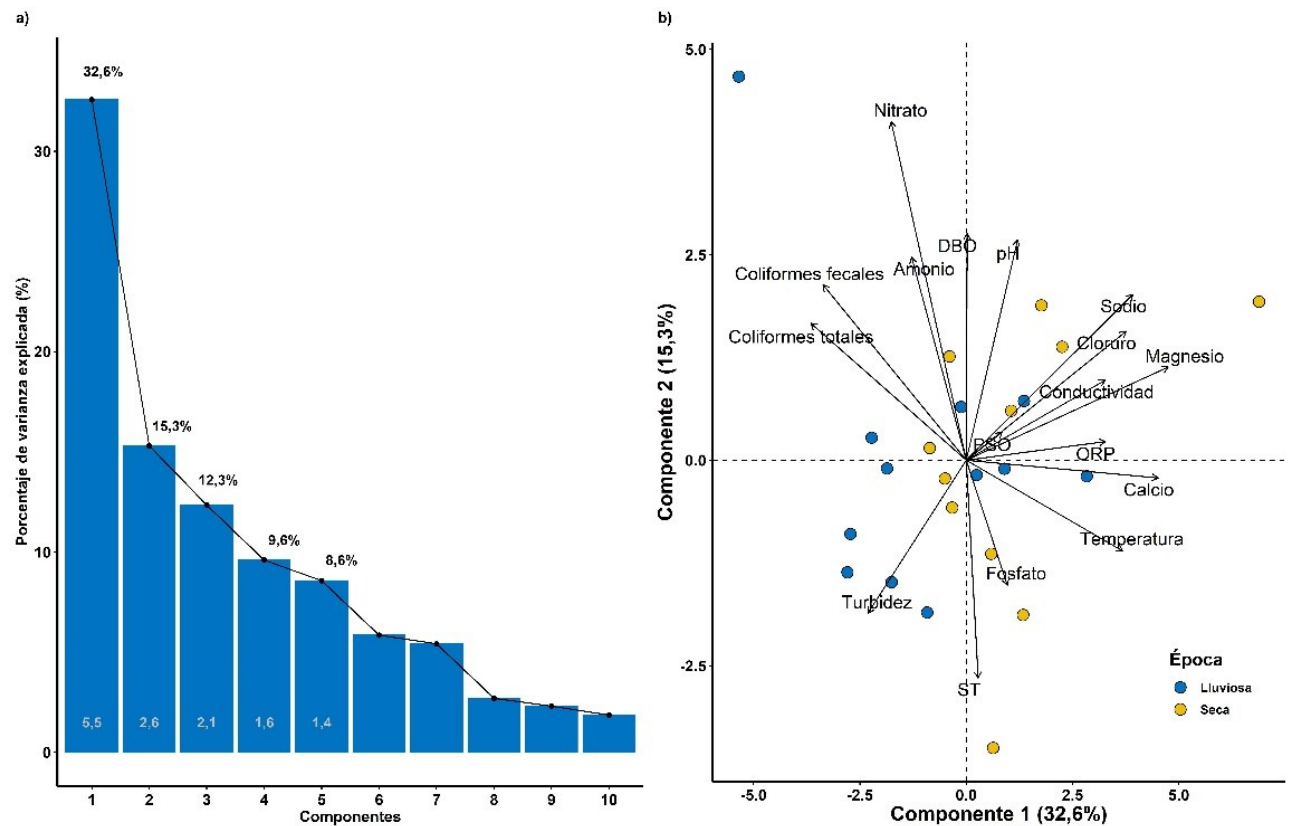

Fig. 2. Análisis de Componentes Principales (a. Componentes principales significativos y varianza explicada, b. Distribución de las variables y sitios según épocas) del Tárcoles, Costa Rica en los años 2017 y 2018.
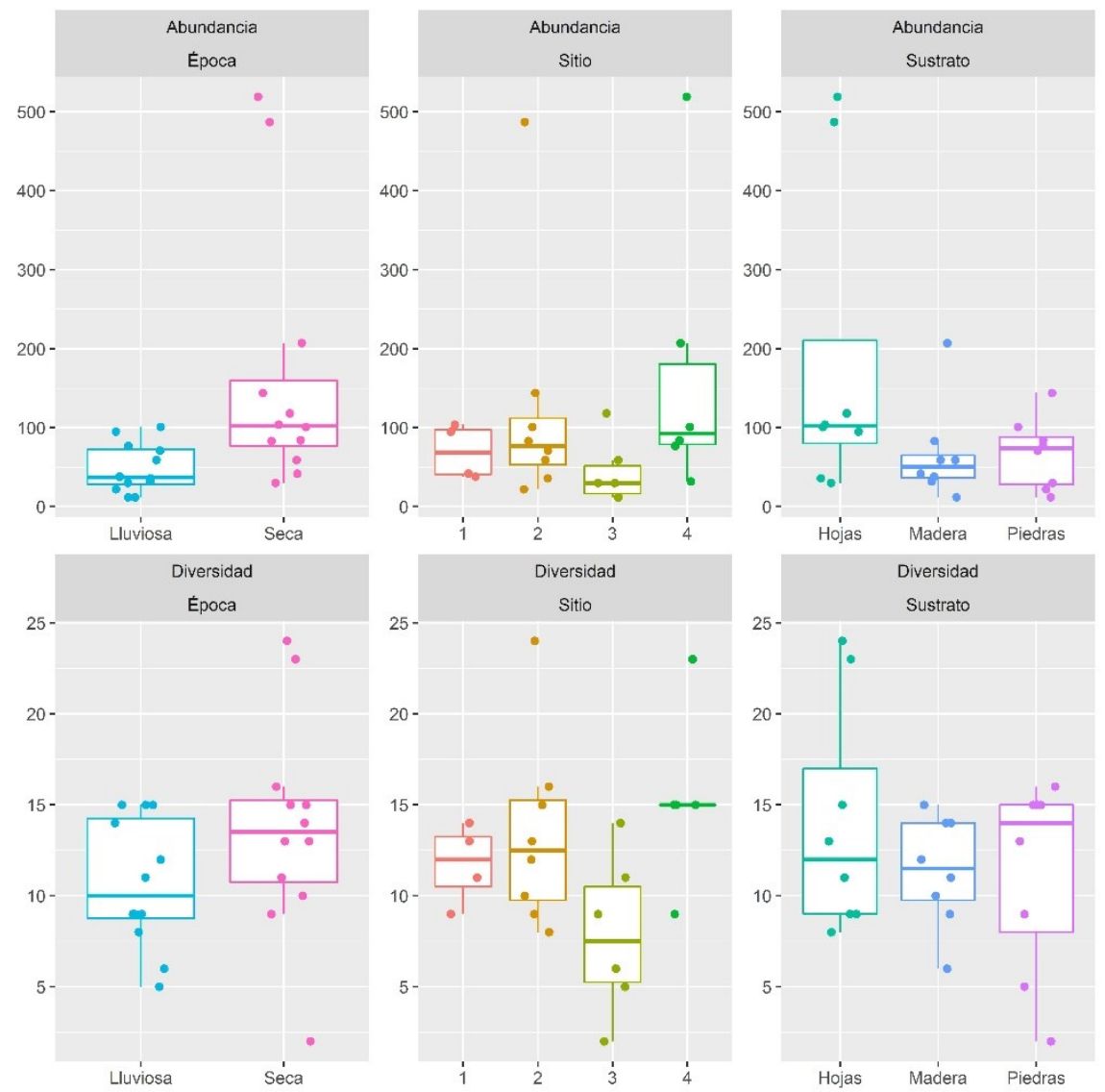

Fig. 3. Abundancia y diversidad de macroinvertebrados acuáticos respecto al sitio de estudio (1: Planta Hidroeléctrica Chucás, 2: Puente de Hamacas, 3: Tajo La Garita, 4: Desembocadura), la época (Lluviosa-Seca) y el sustrato artificial (Hojas, madera, piedras) del Tárcoles, Costa Rica en los años 2017 y 2018. 
Índices de Calidad de Agua (NSF y Holandés): el índice Holandés evidencia que el río presenta contaminación incipiente, excepto el sitio 1 , donde es moderada en época seca; mientras que, el ICA-NSF lo clasifica como un río de calidad media en todos los sitios y en ambas épocas (Fig. 4., Apéndice digital).

\section{DISCUSIÓN}

La cuenca del Tárcoles se caracteriza por un uso de suelo diverso; en la parte baja predominan tierras cultivadas, pasturas y bosque secundario. A pesar de que el urbanismo no es el uso principal, las actividades agropecuarias y de extracción de materiales son constantes a lo largo del cauce. Esto modifica las condiciones físicas y químicas del agua y, por lo tanto, altera la biota acuática (Ongley, 1997; Melland, Fenton, \& Jordan, 2018).

Parámetros físicos, químicos y microbiológicos: Se identificaron fuentes puntuales de contaminación como: desechos industriales, agrícolas, domésticos, entre otros; generados por la alteración del uso de suelo y las actividades antrópicas cercanas al cauce del Tárcoles (Medupin, 2020). El oxígeno en el agua del Tárcoles fue el parámetro con mayores variaciones, y se conoce como uno de los parámetros más importantes en un cuerpo de agua, ya que si se presentan valores inferiores a $5 \mathrm{mg} / \mathrm{L}$ se torna perjudicial para la vida acuática (Calvo-Brenes \& Mora-Molina, 2007; Banjara, Singh, \& Banjara, 2019), así como los valores de PSO inferiores al 55\% aumentan el riesgo de los organismos a sufrir envenenamiento por metales pesados y plaguicidas (Blomquist et al., 2007).

Estas características de anoxia fueron observadas durante la época seca en el sitio 1, donde las condiciones naturales del río han sido intervenidas por la colocación de un embalse, afectando directamente la concentración de OD y PSO, los cuales se mostraron incluso inferiores a los valores mínimos para mantener el equilibrio ecológico de un cuerpo de agua. Además, la temperatura del agua se mostró más baja respecto a los demás sitios, ya que los embalses reducen la amplitud en los cambios diarios de temperatura, que podrían afectar a la fauna acuática (Sabater, Donato, Giorgi, \& Elosegi, 2009). Aunado a lo anterior, en este mismo sitio se presentaron los valores más altos del ion amonio (máximo: 0,862 mg/L, promedio: $0,28 \mathrm{mg} / \mathrm{L}$ ); debido a que, con la actividad metabólica de los microorganismos sobre la materia orgánica del fondo del embalse, no solo se libera más amonio al agua, sino que también se consume el oxígeno presente por la actividad microbiana (USEPA, 2020). Y es así como se observa la interacción entre estos tres parámetros y su resultado principal es el deterioro de las condiciones óptimas y naturales en el cuerpo de agua.

Como ya se ha mencionado, en Costa Rica, la falta de tratamiento de aguas residuales, tanto domésticas como agropecuarias, ocasiona una afectación directa y continua sobre el recurso hídrico (Espinoza \& Villalta, 2004; Chamizo \& Mora, 2006; Calvo-Brenes \& Mora-Molina, 2007; MoralesHidalgo, 2013), lo cual es un factor definitivo de la alteración en las concentraciones de DBO, nitratos y coliformes presentes en el agua del Tárcoles; donde el uso de suelo muestra un cambio abrupto en la parte media del cauce, específicamente en el sito 2 y 3 , permitiendo identificar mayormente en estas zonas fuentes difusas de contaminación, tales como: usos desmedidos de fertilizantes, escorrentía de los campos agrícolas circundantes, pastizales, uso urbano y efluentes industriales (Villegas, Manco, Leclerec, Muller, \& Velásquez, 1995; Mena-Rivera et al., 2018).

El Tárcoles presenta varias canteras y quebradores que durante todo el año se encuentran realizando extracción de materiales y remoción del lecho del cauce; en este caso, el sitio 2 es un quebrador y el sitio 3 es una cantera; estos presentaron los valores más elevados en las concentraciones de ST y turbidez del agua, producto de los efectos erosivos de estas actividades 
(Villegas et al., 1995). Las consecuencias del aumento en la turbidez y los ST en el agua son perjudiciales para muchos organismos acuáticos, reduciendo el éxito reproductivo de muchas especies animales, así como una disminución cuantitativa en el ingreso de la luz, afectando la producción primaria (Melland et al., 2018; Banjara et al., 2019; Windsor, Pereira, Tyler, \& Ormerod, 2019).

Además, estos dos sitios son zonas de pastoreo, donde el ganado ingresa constantemente al agua y genera remoción de material, aumentando la turbidez y el aporte de materia orgánica, lo cual también se asocia con el incremento en las concentraciones de coliformes fecales y totales, que afectaron la presencia de macroinvertebrados acuáticos, registrando la abundancia y diversidad más baja (Dunea et al., 2020); por lo que las buenas prácticas agrícolas como reducir la densidad de ganado o establecer parcelas de pasto permanentes pueden reducir los fosfatos, nitratos y sólidos suspendidos en el agua. Asimismo, establecer coberturas perennes ayuda a reducir los sedimentos, permitiendo que la calidad del agua mejore y a futuro las poblaciones de macroinvertebrados también podrían mostrar una mejoría significativa (Melland et al., 2018).

En el sitio 4, cercano a la desembocadura del Tárcoles hacia el océano pacífico, se presenta una alta mineralización producto de las corrientes marinas que proporcionaron un aumento sustancial en la conductividad, fosfato, cloruro, sodio, magnesio, calcio, potasio, entre otros minerales (El Morhit \& Mouhir, 2014), así como la dilución de los contaminantes, ya que el río se ensancha y aumenta su caudal y podría mostrar un efecto natural de autodepuración (Flores, Ramos, \& Gisberth, 2010).

Parámetros biológicos - Macroinvertebrados: La comunidad biológica depende de los nutrientes en el agua, la época, la altitud, la calidad del agua, los microhábitats, el estado de conservación del sitio, entre otros; ya que existe una relación directa entre los procesos fisiológicos de los macroinvertebrados y los parámetros fisicoquímicos del agua (Banjara et al., 2019; Medupin, 2020). De acuerdo con los resultados, la época seca presentó una mayor abundancia de macroinvertebrados acuáticos, y esto coincide con lo encontrado por Araúz, Amores y Medianero (2000), quienes afirman que, en época lluviosa, la estructura del ambiente lótico y léntico es afectada por la influencia de crecidas y se genera un efecto de arrastre sobre los macroinvertebrados acuáticos, ocurriendo una mayor abundancia de taxones cuando la precipitación es menor; además, en época lluviosa se presentó una mayor carga orgánica, a manera de turbidez que es un factor que puede generar una deflexión en la concentración de OD y a su vez puede llegar a generar una mayor carga microbiana o coliformes fecales, como se evidenció también en este estudio (Orjuela, Saldarriaga, García, \& Wilches, 2010; Suárez, García, \& Vaca, 2012; Goldyn et al., 2018; Windsor et al., 2019; Medupin, 2020; Dunea, et al., 2020).

Con respecto a la abundancia en general de macroinvertebrados acuáticos, la familia Chironomidae fue la más abundante seguida de la subclase Oligochaeta. Los quironómidos son importantes desde el punto de vista cualitativo como cuantitativo, ya que colonizan todo tipo de ambientes acuáticos (Reiss, 1981) y es considerada como la familia más ampliamente distribuida, más diversa y a menudo más abundante de todas las familias de macroinvertebrados bentónicos en los ecosistemas acuáticos (Kranzfelder \& Ferrington, 2018); además, las familias Chironomidae y Psychodidae son indicadoras de aguas mesoeutróficas, mientras que los oligoquetos son comunes en aguas eutroficadas, habitando sobre el fondo fangoso y con abundante cantidad de detritos (Roldán, 1988) lo cual parece haber favorecido su alta abundancia en el ambiente léntico del embalse de la represa hidroeléctrica Chucás (sitio 3).

Un aspecto interesante de recalcar se observó en el sitio 4, donde se dio una aparente mezcla de organismos dulceacuícolas con organismos estuarinos. Se encontraron gasterópodos Hydrobiidae, en donde algunos representantes habitan principalmente en agua dulce o salobre, 
alimentándose de detritos y, algunas veces, en muy altas densidades (Nava, Severeyn \& Machado, 2011). Además, tres taxones más de gasterópodos, Planorbidae, Neritidae y Ostracoda, y un crustáceo, Hyalellidae, fueron recolectados únicamente en dicho sitio. Éstos, excepto Planorbidae, poseen representantes en aguas salobres (Morales-Ramírez \& Jakob, 2008; Blanco-Libreros \& Arroyave-Rincón, 2009; Ríos-Escalante, Morrone, \& Rivera, 2012). Por lo tanto, un posible aumento en la diversidad en este punto podría deberse a la mezcla del ambiente dulceacuícola y marino.

Por otro lado, la abundancia y diversidad de macroinvertebrados acuáticos en los sustratos artificiales no fue significativa en este estudio posiblemente debido, al gran impacto sobre los macroinvertebrados acuáticos, de catástrofes climáticas ocurridas como el Huracán Nate ocurrido en octubre del año 2017. Gracias a datos brindados por los operadores de la Represa Hidroélectrica Chucás, se pudo constatar que este Huracán generó en dicha represa, entre el 05-06 de octubre, un caudal promedio de salida de la presa de $1278,9 \mathrm{~m}^{3} / \mathrm{s}$ por hora. Esto fue casi seis veces más la cantidad que días antes de la tormenta $\left(218,9 \mathrm{~m}^{3} / \mathrm{s}\right.$ por hora el 01 de octubre). Este huracán ocasionó, además, 4211 hectáreas de daños en la producción de la zona (Naranjo, 2017) y lluvias perennes y abundantes hasta enero del 2018 (Alvarado, 2017). A pesar de esto, otros estudios señalan que las densidades de macroinvertebrados son usualmente más altas en paquetes de hojas que en otros sustratos circundante, debido a su importancia como alimento directo e indirecto, en menor medida como refugio e incidentalmente como sustrato del cual aferrarse (Richardson, 1992; Dávila-Recinos, Ortíz-Aldana, \& Reyes-Morales, 2019). Por lo que sería probable que sucediera lo mismo en este cuerpo de agua.

Es importante aclarar que la abundancia de organismos, que no utilicen ninguno de estos tres tipos de sustratos artificiales (madera, piedra, hojas) podría verse subrepresentada. Por lo que, recomendamos en futuros estudios, el uso de dragas bentónicas para complementar la diversidad de macroinvertebrados acuáticos en los sustratos suaves de sedimentos finos del fondo del río.

Índices de Calidad de Agua (NSF y Holandés): La diferencia en los resultados entre los ICA aplicados, demuestran la importancia de utilizar un mayor número de parámetros (físicos, químicos, microbiológicos) en la evaluación de la calidad de los cuerpos de agua. A pesar de que la diferencia entre ambos resultados no es significativa (Mena-Rivera et al., 2018) se observa la tendencia del ICA-NSF a mostrar resultados de aguas con menor calidad, lo anterior como resultado a la incorporación de indicadores microbiológicos y un mayor número de variables físicas y químicas en relación con el índice de calidad Holandés, ya que este último evalúa la calidad en términos del grado de contaminación, únicamente a partir de 3 indicadores fisicoquímicos, limitando la información sobre los procesos de degradación o recuperación que se pudieran suscitar en el ambiente acuático (Samboni et al., 2007).

En términos generales, los resultados de calidad del agua obtenidos son congruentes con estudios publicados por el Plan Nacional para el Monitoreo de la Calidad del Agua Superficial (Ministerio de Ambiente y Energía Dirección de Aguas, 2013), pero en contraste con los resultados de calidad obtenidos para ríos pertenecientes a esta cuenca (Mena- Rivera et al., 2018; Mena-Rivera et al., 2017; Alvarado-García, Pérez-Gómez, \& Gastezzi-Arias, 2020), debido a que se debe tomar en consideración que los sistemas acuáticos poseen la capacidad natural de auto depurarse (Camacho, 2003; Flores et al., 2010), como el caso del Tárcoles, el cual posee características biofísicas (topografía, elevación, lecho pedregoso, volumen de agua, entre otros) que contribuyen con la oxigenación, principalmente mecánica, y la capacidad de autodepuración. Otro aspecto que incide en la recuperación de la calidad del agua del Tárcoles puede estar relacionada en la disminución del urbanismo, en consecuencia, un menor aporte de carga orgánica producto de las aguas residuales que repercuten directamente en el estado de conservación del recurso hídrico (Espinoza \& Villalta, 2004; Chamizo \& Mora, 2006; Calvo-Brenes \& Mora-Molina, 2007). 
Los ICA permiten sintetizar y transmitir los resultados de una forma más simple y clara, permitiendo ser expuestos ante las autoridades de forma funcional en la toma de decisiones en zonas de influencia por contaminantes de origen antropogénico, y así realizar comparaciones entre cuerpos de agua de forma espacial y temporal (Pérez-Castillo \& Rodríguez, 2008). En el caso de la zona de estudio no se observaron diferencias significativas entre épocas y sitios, para ambos índices aplicados; sin embargo, la condición de contaminación moderada en el índice holandés para la época seca en el sitio 1, puede explicarse debido a que en este sitio se encontraron las concentraciones de ion amonio más altas y en época seca se produce un fenómeno de sedimentación de los contaminantes, aumentando los procesos de descomposición, en los cuales se consume mayor cantidad de oxígeno disuelto que ocasiona una escasez de este elemento vital para los organismos acuáticos (Wiik et al., 2015; Wu, Yan, Shang, Shan, \& Wang, 2017).

Finalmente, se evidencia que la contaminación del Tárcoles se ve afectada por diversos factores, como la estacionalidad, prácticas agropecuarias no controladas, presencia secuencial de plantas hidroeléctricas que alteran el curso natural del río y los eventos naturales, son algunas de las causas principales que podríamos señalar. Por lo que, es necesario un plan de gestión integrada de la cuenca, la intervención de sitios prioritarios, el desarrollo de investigaciones orientadas en la salud del ecosistema y la conservación de especies vulnerables, y el uso de herramientas geoespaciales para el monitoreo ambiental de toda la cuenca del Tárcoles.

\section{AGRADECIMIENTOS}

Los autores agradecen a la Universidad Estatal a Distancia y a la Universidad Nacional de Costa Rica por el aporte de recursos en esta investigación; al Sistema Nacional de Áreas de Conservación (SINAC), dependencia del Ministerio de Ambiente y Energía (MINAE) por el otorgamiento de los permisos de recolecta; a la empresa Enel Green Power Costa Rica, específicamente a la Planta Hidroeléctrica Chucás en Atenas, a Jungle Crocodile Safari y al Tajo La Garita por los permisos de ingreso; y a Sergio Quesada Acuña por toda su colaboración.

\section{ÉTICA, CONFLICTO DE INTERESES Y DECLARACIÓN DE FINANCIAMIENTO}

Los autores declaran haber cumplido con todos los requisitos éticos y legales pertinentes, tanto durante el estudio como en el manuscrito; que no hay conflictos de interés de ningún tipo, y que todas las fuentes financieras se detallan plena y claramente en la sección de agradecimientos. Asimismo, están de acuerdo con la versión editada final del documento. El respectivo documento legal firmado se encuentra en los archivos de la revista.

La declaración de la contribución de cada autor al manuscrito es la siguiente: G.P.G., \& V.A.G.: idearon el trabajo; G.P.G., V.A.G., \& F.H.: recolectaron los datos en el campo; R.S.G., F.H., \& J.A.R.R.: procesaron las muestras en laboratorio; G.P.G., \& R.S.G.: realizaron la redacción del manuscrito y análisis de los datos; F.H., V.A.G \& J.A.R.R.: revisaron el manuscrito y realizaron ediciones y sugerencias varias para su mejora. 


\section{REFERENCIAS}

Aho, K. (2014). asbio: A collection of statistical tools for biologists. R Package version, 1-1.

Alvarado, L. F. (2017). Pronóstico Climático Estacional noviembre 2017-enero 2018. Boletín Meteorológico Mensual, 4044.

Alvarado-García, V., Pérez-Gómez, G., \& Gastezzi-Arias, P. (2020). Calidad del ecosistema urbano del río Torres, San José, Costa Rica: factores bióticos y abióticos. UNED Research Journal, 12(2), e3016. DOI: 10.22458/urj.v12i2.3016

APHA., AWWA., \& WEF. (2017). Standard Methods for the Examination of Water \& Wastewater, Standard Methods for the Examination of Water and Wastewater. Washington, DC, EEUU: American Public Health Association.

Araúz, B., Amores, R., \& Medianero, E. (2000). Diversidad y distribución de insectos acuáticos a lo largo del cauce del Río Chico (Provincia de Chiriquí, República de Panamá). Scientia, 15(1), 27-45.

Aznar, A. (2000). Determinación de los parámetros físico-químicos de calidad de las aguas. Gestión Ambiental, 2(23), 1219.

Banjara, B., Singh, R. K., \& Banjara, G. P. (2019). A study on physico chemical parameters of river, urban and rural ponds of Raipur district. International Journal of Development Research, 9(1), 24986-24989.

Bartram, J., \& Ballance, R. (1996). Water quality monitoring: a practical guide to the design and implementation of freshwater quality studies and monitoring programmes. London; New York: E \& FN Spon.

Benavides-Benavides, C., Mena-Rivera, L., Vega-Gúzman, I., Salgado-Silva, V., Coto-Campos, J., Rojas-Cantillano, D., Vargas, Y., \& Rodríguez-Corrales J. A. (2016). Validación de métodos rápidos de análisis para aguas dulces superficiales. Universidad en Diálogo, 6(2), 41-57. DOI: 10.15359/udre.6-2.3

Blanco-Libreros, J. F., \& Arroyave-Rincón, A. (2009). Daños por depredación y tamaño de concha del caracol diádromo Neritina virgínea (Gastropoda: Neritidae) en el río Mameyes, Puerto Rico. Revista de Biología Tropical, 57(4), 1069-1080.

Blanco, K., \& Morera, C. (2013). Recurso hídrico en la zona norte de Heredia: la experiencia de la UNA. Heredia, Costa Rica, Editorial Universidad Nacional.

Blomquist W., Ballestero M., Bhat A., \& Kemper K. E. (2007) Costa Rica: Tárcoles Basin. En: Kemper, K.E., Dinar A., Blomquist W. (eds) Integrated River Basin Management through Decentralization. Heidelberg, Alemania: Springer. DOI: 10.1007/978-3-540-28355-3_8

Brown, R. M., McClelland, N. I., Deininger, R. A., \& Tozer, R. G. (1970). Water quality index-do we dare?. Water Sewage Works, 117(10), 339-343.

Calvo-Brenes, G., \& Mora-Molina, J. (2007). Evaluación y clasificación preliminar de la calidad del agua de la cuenca del río Tárcoles y el Reventazón Parte I: Análisis de la contaminación de cuatro ríos del área metropolitana. Tecnología en Marcha, 20(2), 3-9.

Castillo, M., Coto, J. M., \& Herrera, J. (2012). Desechos líquidos: el caso de las salas de belleza. Comunicación preliminar. Uniciencia, 26(1-2), 23-27.

Camacho, L.A. (2003). Modelación de la Calidad del Agua del Río Grande de Tárcoles, Costa Rica. Informe final. Instituto Costarricense de Acueductos y Alcantarillados-Organización Panamericana de la Salud. San José, Costa Rica.

Chamizo, H. A., \& Mora, D. A. (2006). Estudio ecológico de las enfermedades de transmisión hídrica en la Cuenca Hidrográfica superficial del Río Grande de Tárcoles. Revista Costarricense de Salud Pública, 15(29), 8-24.

Coto, J. M., Benavides, A. C., \& Segura, R. (2008). El papel de la escuela primaria en la gestión ambiental comunitaria en Las Vueltas de La Guácima, Alajuela. Biocenosis, 21(1-2), 1-6. 
Dávila-Recinos, G., Ortíz-Aldana, J. R., \& Reyes-Morales, F. (2019). Efecto del microhábitat sobre la abundancia y riqueza específica de los macroinvertebrados bentónicos en dos ríos tropicales de montaña, Guatemala. Ciencia, Tecnología y Salud, 6(1), 7-21.

División de Fiscalización Operativa y Evaluativa (DFOE). (2013). Informe acerca de la eficacia del estado para garantizar la calidad del agua en sus diferentes usos. San José, Costa Rica, Área de Servicios Ambientales y de Energía de la $\begin{array}{llll}\text { Contraloría General del Recuperado de } & \end{array}$ https://cgrfiles.cgr.go.cr/publico/docs_cgr/2013/SIGYD_D_2013003909.pdf

Dunea, D., Bretcan, P., Tanislav, D., Serban, G., Teodorescu, R., lordache, S., Petrescu, N., \& Tuchiu, E. (2020). Evaluation of Water Quality in Ialomita River Basin in Relationship with Land Cover Patterns. Water, 12, 735. DOI: 10.3390/w12030735

Espinoza, C. E., \& Villalta, R. A. (2004). Primera etapa del Plan de Manejo Integral del Recurso Hídrico: la estrategia nacional para la GIRH en Costa Rica (ATNIWP 8467-CR). Estudio de caso sobre la contaminación de la Cuenca de los ríos Virilla y Grande de Tárcoles (Cuenca 24). San José, Costa Rica.

El Morhit, M., \& Mouhir, L. (2014). Study of phisyco-chemical parameters of water in the Loukkos river estuary (Larache, Morocco). Environmental Systems Research, 3(17), 1-9.

Forero-Céspedes, A., Reinoso-Flórez, G., \& Gutiérrez, C. (2013). Evaluación de la calidad del agua del río Opia (TolimaColombia) mediante macroinvertebrados acuáticos y parámetros fisicoquímicos. Caldasia, 35(2), 371-387.

Flores B, M. L., Ramos R, O., \& Gisberth, E. (2010). Evaluación de la contaminación antrópica de las aguas del río Jillusaya. Revista Boliviana de Química, 27(2), 75-80.

Gastezzi-Arias, P., Alvarado-García, V., \& Pérez-Gómez, G. (2017). La importancia de los ríos como corredores interurbanos. Biocenosis 31(1-2): 39-45.

Goldyn, R., Szpakowska, B., Swierk, D., Domek, P., Buxakowski, J., Dondajewska, R., Baralkiewicz, D., \& Sajnóg, A. (2018). Influence of stormwater runoff on macroinvertebrates in a small urban river and a reservoir. Science of the Total Environment, 625, 743-751. DOI: 10.1016/j.scitotenv.2017.12.324

Gurdián, R., \& Coto, J. M. (2010). Estudio preliminar del uso de la semilla de tamarindo (Tamarindus indica) en la coagulación - floculación de aguas residuales. Tecnología en Marcha, 24(2), 18-26.

Helsel, D. R. (2012). Statistics for Censored Environmental Data Using Minitab an R. New Jersey, United States of America: John Wiley \& Sons, Inc. All.

Herrera, J., Rodríguez, S., Rojas, J.F., Herrera, E., \& Chaves, M. (2013). Variación temporal y espacial de la calidad de las aguas superficiales en la subcuenca del río Virilla (Costa Rica) entre 2006 y 2010. Revista de Ciencias Ambientales, 45(1), 51-62.

Kassambara, A. (2015). Factoextra: Visualization of the outputs of a multivariate analysis. R Package version, 1(1), 1-75.

Kranzfelder, P., \& Ferrington, L. C. (2018). Chironomidae (Diptera) species diversity of stuaries across a land use gradient on the Caribbean coast of Costa Rica. Revista de Biología Tropical, 66(3), 1118-1134. DOI: $10.15517 /$ rbt.v66i3.31927

Kruskal, W. H., \& Wallis, W. A. (1952). Use of ranks in one-criterion variance analysis. Journal of the American statistical Association, 47(260), 583-621.

La Gaceta. (2007). Reglamento para la Evaluación y Clasificación de la Calidad de Cuerpo de Agua Superficiales № 33903MINAE-S. San José, Costa Rica: Imprenta Nacional.

Lee, L. (2017). NADA: Nondetects and Data Analysis for Environmental Data. R Package version, 1.6-1.1. Recuperado de https://CRAN.R-project.org/package=NADA 
Lozano, L. (2005). La bioindicación de la calidad del agua: importancia de los macroinvertebrados en la cuenca alta del río Juan Amarillo, Cerros Orientales de Bogotá. Umbral Científico, 7, 5-11.

Mauger, A. L., Velez, E., Cherkiss, M. S., Brien, M. L., Boston, M., Mazzotti, F. J., \& Spotila, J. R. (2012). Population assessment of the American crocodile, Crocodylus acutus (Crocodilia: Crocodylidae) on the Pacific coast of Costa Rica. Revista de Biología Tropical, 60(4), 1889-1901.

Medupin, C. (2020). Spatial and temporal variation of benthic macroinvertebrate communities along an urban river in Greater Manchester, UK. Environmental Monitoring and Assessment, 192(84), 1-20. DOI: 10.1007/s10661-0198019-6

Melland, A. R., Fenton, O., \& Jordan, P. (2018). Effects of agricultural land management changes on surface water quality: A review of meso-scale catchment research. Environmental Science and Policy, 84, 19-25. DOI: 10.1016/j.envsci.2018.02.011

Meléndez, E., \& Russo, R. O. (Noviembre del 2016). Abordaje Integral a la solución de los problemas hídricos que afectan a la Cuenca del Río Grande de Tárcoles, Costa Rica, según la resolución № 5894-2007, del Tribunal Constitucional. En Rodríguez-Mateo, J. R. (Ed.). Centroamérica Agua, cultura y territorio. Actas del I Congreso Internacional. Congreso llevado a cabo en Heredia, Costa Rica.

Mena-Rivera, L., Salgado-Silva, V., Benavides-Benavides, C., Coto-Campos, J. M., \& Swinscoe, T. H. A. (2017). Spatial and seasonal surface water quality assessment in a tropical urban catchment: Burío River, Costa Rica. Water (Switzerland), 9(8). DOI: 10.3390/w9080558

Mena-Rivera, L., Vásquez-Bolaños O., Gómez-Castro, C., Fonseca-Sánchez, A., Rodríguez-Rodríguez A., \& SánchezGutiérrez, R. (2018). Ecosystemic Assessment of Surface Water Quality in the Virilla River: Towards Sanitation Processes in Costa Rica. Water, 10(7), 845. DOI: 10.3390/w10070845

Merck, E. (2005). Microbiology Manual, Fluorocult ${ }^{\circledR}$ LMX Broth Modified. Darmstadt, Germany: Merck.

Merritt, R. W., Cummins, K. W., \& Berg, M. (2008). An introduction to the aquatic insects of North America. lowa, United states of America: Kendall/Hunt Publishing Company.

Ministerio de Ambiente y Energía Dirección de Aguas. (2013). Plan Nacional de Monitoreo de la Calidad de los Cuerpos de Agua Superficiales. Recuperado de http://www.da.go.cr/wp-content/uploads/2016/05/Plan-de-Monitoreo-deAguas-Superficiales-FINAL-Mayo-2013.pdf

Morales-Hidalgo, D. (2013). Capítulo 6 Costa Rica. En Mahlknecht, J. \& Pastén Zapata, E. (Coords). Diagnóstico de los recursos hídricos en América Latina, (pp. 221-252). Juárez, México: Pearson.

Morales-Ramírez, A., \& Jakob, J. (2008). Seasonal vertical distribution, abundance, biomass, and biometrical relationships of ostracods in Golfo Dulce, Pacific coast of Costa Rica. Revista de Biología Tropical, 56(4), 125-147.

Nava, M., Severeyn, H., \& Machado, N. (2011). Distribución y taxonomía de Pyrgophorus platyrachis (Caenogastropoda: Hydrobiidae), en el Sistema de Maracaibo, Venezuela. Revista de Biología Tropical, 59(3), 1165-1172.

Naranjo, J. D. (2017). Resumen Meteorológico de octubre 2017. Boletín Meteorológico Mensual, 2-39.

Ongley, E. D. (1997). Lucha Contra la Contaminación Agrícola de los Recursos Hídricos. (Estudio FAO Riego y Drenaje - 55). Italia: FAO.

Oram, B. (2013). Monitoring the Quality of Surface Waters (WQI Calculator). Recuperado de: https://waterresearch.net/index.php/water-treatment/water-monitoring/monitoring-the-quality-of-surfacewaters

Orjuela, C., Saldarriaga, G., Garcia, M., \& Wilches, H. (2010). Capítulo 6: Calidad del agua superficial en Colombia. Colombia: IDEAM 
Pastor, A., Varela, P., Bianchi, P., \& Durando, P. (2015). Calidad Bacteriológica del agua del río San Juan en zonas aledañas a la desembocadura del arroyo los Tapones (San Juan, Argentina). Natura Neotropicalis, 46(1), 7-46.

Pérez, C., Coto, J. M., Salgado, V., Herrera, J., Fernández, J. F., \& Benavides, C. (2012). Tratamiento de aguas residuales con tecnologías alternativas en una pequeña unidad doméstica-productiva. Uniciencia, 27(1), 319-331.

Pérez-Castillo, A. G., \& Rodríguez, A. (2008). Índice fisicoquímico de la calidad de agua para el manejo de lagunas tropicales de inundación. Revista de Biología Tropical, 56(4), 1905-1918.

Pérez-Gómez, G., Jiménez-Rocha, A. E., \& Bermúdez-Rojas, T. (2018). Parásitos gastrointestinales de aves silvestres en un ecosistema urbano tropical en Heredia, Costa Rica. Revista de Biología Tropical, 66(2), 788-798. DOI: 10.15517/rbt.v66i2.33409

Pérez, D. J., Segovia, J. A., Cabrera, P. C., Delgado, I. A. \& Martins, M. L. (2018). Uso del suelo y su influencia en la presión y degradación de los recursos hídricos en cuencas hidrográficas. Revista de Investigación Agraria y Ambiental, 9(1), 41-57. DOI: 10.22490/21456453.2089

Peto, R., \& Peto, J. (1972). Asymptotically Efficient Rank Invariant Test Procedures. Journal of the Royal Statistical Society. Series A (General), 135(2), 185-207. DOI: 10.2307/2344317

Prat, N., Ríos, B., Acosta, R., \& Rieradevall, M. (2009). Los macroinvertebrados como indicadores de calidad de las aguas En E. Domínguez, \& H. R. Fernández (eds.). Macroinvertebrados bentónicos sudamericanos. Sistemática y biología (pp. 631-654). Tucumán, Argentina: Fundación Miguel Lillo.

Prentice, R., \& Marek, P. (1979). A Qualitative Discrepancy between Censored Data Rank Tests. Biometrics, 35(4), 861-867. Recuperado de https://www.jstor.org/stable/2530120

Presidencia de la República. (2016). Planta de tratamiento los Tajos genera beneficios adicionales al saneamiento. San José, Costa Rica. Recuperado de https://www.presidencia.go.cr/comunicados/2016/03/planta-de-tratamientolos-tajos-genera-beneficios-adicionales-al-saneamiento/

Quesada, M. (2012). Dinámica territorial en el uso de la tierra y el régimen hidrológico: región central, Costa Rica. Espacio y Desarrollo, (24), 45-56.

Quesada-Alvarado, F., \& Solano-Ulate, D. (2020). Colonización de macroinvertebrados acuáticos en tres tipos de sustratos artificiales, en un río tropical. Revista de Biología Tropical, 68(2), 68-78.

R Development Core Team (2020). The R Project for statistical computing. Recuperado de: https://www.r-project.org/

Ramírez, A. (2010). Métodos de Recolecta. Revista de Biología Tropical, 58(4), 41-50.

Reiss, F. (1981). Chironomidae. En S. H. Hurlbert, G. Rodríguez, \& N. D. Dos Santos (Eds). Aquatic Biota of Tropical South America, Part 1, Arthropoda. (pp. 261-268). San Diego, Estados Unidos: Universidad de San Diego.

Richardson, J. S. (1992). Food, microhábitat, or both? Macroinvertebrate use of leaf accumulations in a montane stream. Freshwater Biology, 27, 169-176.

Ríos-Escalante, P., Morrone, J. J., \& Rivera, R. (2012). Patrones de distribución de especies sudamericanas de Hyalella (Amphipoda: Hyalellidae). Gayana, 76(2), 153-161.

Roldán, G. (1988). Guía para el estudio de los Macroinvertebrados Acuáticos del Departamento de Antioquia, Fondo FENColombia. Bogotá, Colombia: Presencia S. A.

Sabater, S., Donato, J. C., Giorgi, A., \& Elosegi, A. (2009). El río como sistema. En A. Elosegi, \& S. Sabater, (Eds). Conceptos y técnicas en ecología fluvial, (pp. 1-17), Salamanca, Madrid: Fundación BBVA.

Samboni, N., Carvajal, Y., \& Escobar, J. C. (2007). Revisión de parámetros fisicoquímicos como indicadores de calidad y contaminación del agua. Revista Ingeniería e Investigación, 27(3), 172-181. 
Sánchez, A. (2017). CostaRicaBosque17. [Costa Rica] [Formato TIFF]. 30x30m de píxel. Proyección CRTM05. Clasificación de la cobertura de la tierra para Costa Rica en el año 2017. Canadá. Centre for Earth Observation Sciences.

Springer, M. (2010). Biomonitoreo acuático. Revista de Biología Tropical, 58(suppl. 4), 53-59.

Springer, M., Ramírez, A., \& Hanson, P. (2010). Macroinvertebrados de agua dulce de Costa Rica I. Revista de Biología Tropical, 58(4), 1-200.

Suárez, A. F., García, C. A., \& Vaca, M. L. (2012). Identificación y evaluación de la contaminación del agua por curtiembres en el municipio de Villapinzón. Tecnura, 16, 185-193.

United States Environmental Protection Agency (USEPA). (2020). Causal Analysis/Diagnosis Decision Information System (CADDIS): Ammonia, Retrieved. Washington, DC, United States of America: Office of Research and Development. Recuperado de: https://www.epa.gov/caddis-vol2/ammonia

Vargas, M. (1974). Llave gráfica para la identificación de larvas de las familias más comunes de dípteros acuáticos, O’Bios, 2(7), 17-38.

Villegas, J. G., Faustino J., Leclerec, G., Muller, S., \& Velásquez, S. (1995). Evaluación de la calidad del agua en la cuenca del río Reventado, Cartago, Costa Rica bajo el enfoque de indicadores de sostenibilidad. Semana Científica, 2, 128-131. Turrialba, Costa Rica.

Wickham, H. (2016). Ggplot2: Elegant Graphics for Data Analysis. Springer-Verlag New York. ISBN 978-3-319-24277-4. Recuperado de: http://ggplot2.tidyverse.org

Windsor, F. M., Pereira, M. G., Tyler, C. R., \& Ormerod, S. J. (2019). Persistent contaminants as potential constraints on the recovery of urban river food webs from gross pollution. Water Research, 163, 114858. DOI: $10.1016 /$ j.watres.2019.114858

Wiik, E., Bennion, H., Sayer, C. D., Davidson, T. A., Mcgowan, S., Patmore, I. R., \& Clarke, S. J. (2015). Ecological Sensitivity of Marl Lakes to Nutrient Enrichment: Evidence from Hawes Water, UK. Freshwater Biology, 60(11), 2226-2247. DOI: $10.1111 /$ fwb.12650

Woomer, P. L. (1994). Most probable number counts. Methods of Soil Analysis: Part 2. Microbiological and Biochemical Properties, 5, 59-79.

Wu, D., Yan, H., Shang, M., Shan, K., \& Wang, G. (2017). Water Eutrophication Evaluation Based on Semi-Supervised Classification: A Case Study in Three Gorges Reservoir. Ecological indicators, 81, 362-372. DOI: 10.1016/j.ecolind.2017.06.004

Zhen-Wu, B. (2010). Índices de calidad del agua en la microcuenca de la quebrada Victoria, Guanacaste, Costa Rica (20072008). UNED Research Journal, 2(1), 45-61. DOI: 10.22458/urj.v2i1.221

\section{EDITADO POR JULIÁN MONGE-NÁJERA}

